\title{
Self-consistent account for phonon induced corrections to quadrupole moments of odd nuclei. Pole and non-pole dia- grams.
}

\author{
E. E. Saperstein ${ }^{1}$, 2,a , S. Kamerdzhiev ${ }^{1}$, D. S. Krepish ${ }^{1}$, S. V. Tolokonnikov ${ }^{1}{ }^{3}$, and D. Voitenkov ${ }^{4}$ \\ ${ }^{1}$ National Research Centre "Kurchatov Institute", 123182, Moscow, Russia \\ ${ }^{2}$ National Research Nuclear University MEPhl, 115409 Moscow, Russia \\ ${ }^{3}$ Moscow Institute of Physics and Technology, 141700, Dolgoprudny, Moscow Region, Russia \\ ${ }^{4}$ Institute for Physics and Power Engineering, 249033 Obninsk, Russia
}

\begin{abstract}
Recent results of the description of quadrupole moments of odd semi-magic nuclei are briefly reviewed. They are based on the self-consistent theory of finite Fermi systems with account for the phonon-particle coupling (PC) effects. The self-consistent model for describing the PC effects was developed previously for magnetic moments. Account for the non-pole diagrams is an important ingredient of this model. In addition to previously reported results for the odd In and Sb isotopes, which are the proton-odd neighbors of even tin nuclei, we present new results for odd $\mathrm{Bi}$ isotopes, the odd neighbors of even lead isotopes. In general, account for the PC corrections makes the agreement with the experimental data significantly better.
\end{abstract}

\section{Introduction}

Spartak Belyaev was one the creators of the microscopic nuclear physics. His famous article of 1959 [1] contained several cornerstones of modern nuclear theory. A crucial role of the first $2^{+}$excitations in even-even spherical nuclei, the quadrupole "phonons", is one of them. The quadrupole phonons are the surface vibrations, belonging to the Goldstone branch related to the spontaneous breaking of the translation symmetry in nuclei. They play the main role in the problem of the phonon-particle coupling (PC) corrections to characteristics of the ground states of odd nuclei we consider.

Consideration of such effects within the self-consistent theory of finite Fermi systems (TFFS) [2] or any other approach based on the use of phenomenological parameters is a rather delicate problem as these parameters could include implicitly some of these effects. In principle, two strategies can be used. The first one was chosen in the self-consistent TFFS [2]. This approach is based on the general principles of the TFFS [3] supplemented with the TFFS self-consistency relation [4]. In this method, the parameters from the beginning were chosen so to describe the nuclear characteristics with account for the PC contributions. Another way was chosen by Fayans with coauthors who developed the energy density functional (EDF) method [5-8] supposing that the EDF parameters include all the PC effects on average. Indeed, the Fayns EDFs FanDF ${ }^{0}$ [7] or DF3 [6, 8] described the binding energies and radii of spherical nuclei with rather high accuracy at the mean field level. Later, again

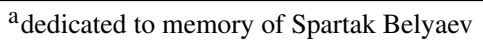


at the mean field level, the EDF DF3 and its version DF3-a [9] with changed spin-orbit and effective tensor parameters, were successfully applied to describe magnetic $[10,11]$ and quadrupole [12-14] moments of odd spherical nuclei. The characteristics of the first $2^{+}$levels in even $\mathrm{Sn}$ and $\mathrm{Pb}$ isotopes $[12,15]$ were also described perfectly well, much better than in the analogous calculations [16] with the use of the Skyrme EDFs SLy4 and SkM*. The list of successful applications of the Fayans EDFs to spherical nuclei can be supplemented with description of the single-particle (SP) spectra of magic nuclei [17]. Recently, the Fayans method with the $\mathrm{EDF} \mathrm{FaNDF}^{0}$ was developed for deformed nuclei [18]. The first applications of this EDF in this field [19-23] turned out to be rather successful.

However, there are PC corrections to characteristics of odd nuclei which behave in a non-regular way depending on the nucleus under consideration and on the SP state $|\lambda\rangle$ of the odd nucleon. At first, it concerns the $\mathrm{PC}$ corrections induced by the $2_{1}^{+}$phonons which are, as a rule, the lowest excitations of the even-even spherical nuclei. It occurs because some PC corrections to different nuclear characteristics depend on the $L$-phonon excitation energy $\omega_{L}$ at small $\omega_{L}$ values as $1 / \omega_{L}$ [2]. The excitation energy of the $2_{1}^{+}$phonon behaves usually in a non-regular way, especially in vicinity of magic nuclei. For example, in the lead isotopic chain we have $\omega_{2} \simeq 4 \mathrm{MeV}$ in ${ }^{208} \mathrm{~Pb}$ and $\omega_{2}<1 \mathrm{MeV}$ in all lighter even $\mathrm{Pb}$ isotopes. Evidently, it is practically impossible to describe so non-regular behavior of the PC corrections with a universal set of the EDF parameters. Thus, if we want to reach higher accuracy in reproducing nuclear data, we should try to separate some fluctuating part of the PC corrections in order to add them to the mean field predictions. Such a programme was carried out for SP levels in magic [17] and semi-magic [27] and for the double odd-even mass differences of magic [28, 29] and semi-magic [30] nuclei. In all the cases, inclusion of the PC corrections made agreement with the data better.

In Refs. [24-26], a model was developed to find the fluctuating part of the PC corrections to magnetic moments of odd semi-magic nuclei found previously within the mean field theory [10, 11]. A semi-magic nucleus contains two subsystems with absolutely different properties. One of them is superfluid, whereas the second subsystem is normal. The model under discussion was developed for nuclei with the odd nucleon belonging to the normal sub-systems. It simplifies the formulas for the PC corrections drastically. Recently, a similar model was developed for the quadrupole moments of odd semi-magic nuclei [31]. The odd-proton neighbors of the even tin nuclei, i.e. the odd isotopes of In and $\mathrm{Sb}$, were considered.

In Sect. 2, we describe briefly the model under discussion, for the case of quadrupole moments. In Sect. 3 we present briefly the results of [31] for the PC corrections, induced by the $2_{1}^{+}$phonons, to quadrupole moments of odd In and $\mathrm{Sb}$ isotopes. The new results of similar consideration for the odd $\mathrm{Bi}$ isotopes are given in Sect. 4. At last, Sect. 5 contains main our conclusions.

\section{The model for PC corrections to multipole moments}

In this Section, we present briefly the formalism used, for the case of quadrupole moments. For magnetic moments, all formulas are similar and can be found in [24, 26]. Within the TFFS [3], quadrupole moments of odd nuclei are determined in terms of the diadonal matrix elements

$$
Q_{\lambda}=\langle\lambda|V| \lambda\rangle_{m=j}
$$

$|\lambda\rangle$ being the state of the odd nucleon, of the normal component $V$ of the effective field. In magic nuclei the latter obeys the RPA-like equation,

$$
V=V_{0}+\mathcal{F} A V,
$$



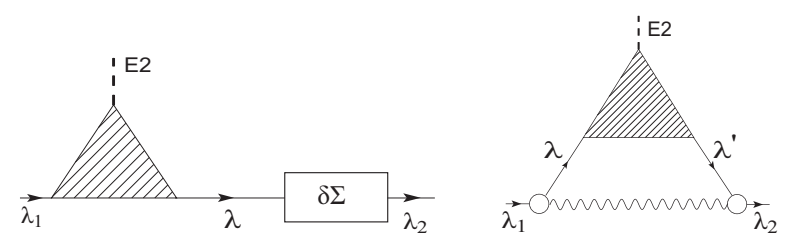

Figure 1. Diagrams for two main PC corrections to the quadrupole moment of an odd nucleus: the "end correction" (left) and the one due to the induced interaction (right).

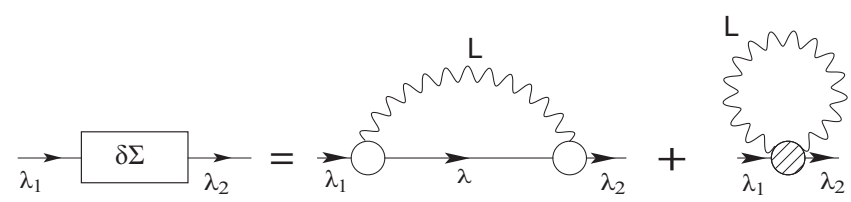

Figure 2. $g_{L}^{2}$ phonon corrections to the mass operator. The dashed blob denotes the sum of non-pole ("phonon tadpole") diagrams.

where $V_{0}$ is the external field, $\mathcal{F}$ is the Landau-Migdal interaction amplitude, and $A$ is the particlehole propagator. For the quadrupole moment problem we discuss, one has $V_{0}(\mathbf{r})=\sqrt{16 \pi / 5} r^{2} Y_{20}(\mathbf{n})(1+$ $\left.\tau_{3}\right) / 2$. In fact, we deal with superfluid nuclei, and the RPA eq. (2) should be replaced with the analogous QRPA equation which can be written symbolically in the same form, but all the terms are now matrices. The explicit form of this equation can be found in [3] or, for the case of the Fayans EDF, which we use as a generator of the self-consistent basis, in [12].

Let us now consider the PC corrections to the matrix element (1) in the field of the $L$-phonon, the phonon creation amplitude being $g_{L}(\mathbf{r}):|\lambda\rangle \rightarrow|\tilde{\lambda}\rangle, V_{0} \rightarrow \tilde{V}_{0}, V \rightarrow \tilde{V}, \mathcal{F} \rightarrow \tilde{\mathcal{F}}$, and $A \rightarrow \tilde{A}$, with obvious notation. We follow the scheme of [24] and limit ourselves to the $g_{L}^{2}$-approximation. We address to $[24,26]$ for the detailed formalism. All the PC corrections we consider are illustrated with the diagrams depicted on Figs. $1-3$. Usual graphic elements are used: the solid line denotes the Green function $\mathrm{G}$, the open circle, the vertex $g_{L}$, the dashed triangle is the effective field $V$, whereas the wavy line denotes the phonon $D$-function:

$$
D_{L}(\omega)=\frac{1}{\omega-\omega_{L}+i \gamma}-\frac{1}{\omega+\omega_{L}-i \gamma} .
$$

As it was written above, the odd nucleon, the state $|\lambda\rangle$ in (1), belongs to the normal subsystem of the semi-magic nucleus under consideration. This makes the problem of finding the PC corrections rather simple. For a superfluid system, an analog of each of the diagrams in Figs. 1 and 2 looks much more complicated [32].

In magic nuclei, the $L$-phonon creation amplitude $g_{L}$ obeys the homogeneous counterpart of Eq. (2):

$$
g_{L}(\omega)=\mathcal{F} A(\omega) g_{L}(\omega)
$$

In a semi-magic one, just as in the case of (2), a matrix QRPA-like analog of this equation appears, $g \rightarrow \hat{g}_{L}$, where $\hat{g}_{L}$ is the 3 -vector containing the normal component $g_{L}^{(0)}$ and two anomalous ones, $g_{L}^{(1,2)}$. As it was demonstrated in $[12,13]$, the normal component is important only in the problem under consideration. So, the anomalous ones will be neglected and the upper index will be omitted 
for brevity, $g_{L}^{(0)} \rightarrow g_{L}$, in all the formulas below. All the low-lying phonons we will consider have natural parity, hence the vertex $g_{L}$ possesses even $T$-parity. It is a sum of two components with spins $S=0$ and $S=1$, respectively:

$$
g_{L}=g_{L 0}(r) T_{L L 0}(\mathbf{n}, \alpha)+g_{L 1}(r) T_{L L 1}(\mathbf{n}, \alpha),
$$

where $T_{J L S}$ stand for the usual spin-angular tensor operators [33]. The operators $T_{L L 0}$ and $T_{L L 1}$ have opposite $T$-parities, hence the spin component should be an odd function of the excitation energy, $g_{L 1} \propto \omega_{L}$. In all the cases we consider, the $2_{1}^{+}$phonon excitation energy is small, $\omega_{2}<1 \mathrm{MeV}$, and the $S=1$ component can be neglected.

Let us begin from the "end correction", the left diagram in Fig. 1. Evidently, the analogous diagram there is, where the left end is PC-corrected. For the diagonal matrix element (1) of the effective field, the sum of these corrections corresponds to the following formula:

$$
\begin{aligned}
\delta V_{\lambda \lambda}^{\mathrm{end}} & =-\sum_{\lambda^{\prime}} V_{\lambda \lambda^{\prime}} G_{\lambda^{\prime}}\left(\varepsilon_{\lambda}\right) \delta \Sigma_{\lambda^{\prime} \lambda}\left(\varepsilon_{\lambda}\right) \\
& -\sum_{\lambda^{\prime}} \delta \Sigma_{\lambda \lambda^{\prime}}\left(\varepsilon_{\lambda}\right) G_{\lambda^{\prime}}\left(\varepsilon_{\lambda}\right) V_{\lambda^{\prime} \lambda}
\end{aligned}
$$

where

$$
G_{\lambda}(\varepsilon)=\frac{n_{\lambda}}{\varepsilon-\varepsilon_{\lambda}-i \gamma}+\frac{1-n_{\lambda}}{\varepsilon-\varepsilon_{\lambda}+i \gamma} .
$$

Two $g_{L}^{2}$ diagrams for the PC correction $\delta \Sigma_{L}$ are depicted on Fig. 2. The second, non-pole term of $\delta \Sigma$ is rather essential in the problem of the PC corrections to the single-particle levels [17, 27], but it is not so in the problem under consideration [24, 26, 31], therefore we do not consider its explicit form here.

On the contrary, the pole term is of primary importance, therefore we write down the corresponding explicit expression, although it is well-known:

$$
\begin{aligned}
\delta \Sigma_{\lambda_{2} \lambda_{1}}^{\text {pole }}(\epsilon) & =\sum_{\lambda M}\left\langle\lambda_{2}\left|g_{L M}^{+}\right| \lambda\right\rangle\left\langle\lambda\left|g_{L M}\right| \lambda_{1}\right\rangle \\
& \times\left(\frac{n_{\lambda}}{\varepsilon+\omega_{L}-\varepsilon_{\lambda}}+\frac{1-n_{\lambda}}{\varepsilon-\omega_{L}-\varepsilon_{\lambda}}\right),
\end{aligned}
$$

where $n_{\lambda}=(0,1)$ stands for the occupation numbers.

After substitution of (8) into (6) one obtains:

$$
\begin{aligned}
\delta V_{\lambda \lambda}^{\text {end }} & =-\sum_{\lambda_{1} \lambda_{2} M} \frac{V_{\lambda_{1}}\left\langle\lambda\left|g_{L M}^{+}\right| \lambda_{2}\right\rangle\left\langle\lambda_{2}\left|g_{L M}\right| \lambda_{1}\right\rangle}{\varepsilon_{\lambda}-\varepsilon_{\lambda_{1}}} \\
& \times\left(\frac{n_{\lambda_{2}}}{\varepsilon_{\lambda}+\omega_{L}-\varepsilon_{\lambda_{2}}}+\frac{1-n_{\lambda_{2}}}{\varepsilon_{\lambda}-\omega_{L}-\varepsilon_{\lambda_{2}}}\right) \\
& -\sum_{\lambda_{1} \lambda_{2} M}\left(\frac{n_{\lambda_{2}}}{\varepsilon_{\lambda}+\omega_{L}-\varepsilon_{\lambda_{2}}}+\frac{1-n_{\lambda_{2}}}{\varepsilon_{\lambda}-\omega_{L}-\varepsilon_{\lambda_{2}}}\right) \\
& \times \frac{\left\langle\lambda\left|g_{L M}^{+}\right| \lambda_{2}\right\rangle\left\langle\lambda_{2}\left|g_{L M}\right| \lambda_{1}\right\rangle V_{\lambda_{1} \lambda}}{\varepsilon_{\lambda}-\varepsilon_{\lambda_{1}}} .
\end{aligned}
$$

In this equation, the terms with $\lambda_{1}=\lambda$ in both the sums are singular. This singularity is removed with the standard renormalization $[2,3]$ of the single particle wave functions $|\lambda\rangle \rightarrow \sqrt{Z_{\lambda}}|\lambda\rangle$, where

$$
Z_{\lambda}=\left(1-\left.\frac{\partial \delta \Sigma_{\lambda \lambda}(\varepsilon)}{\partial \varepsilon}\right|_{\varepsilon=\varepsilon_{\lambda}}\right)^{-1}
$$



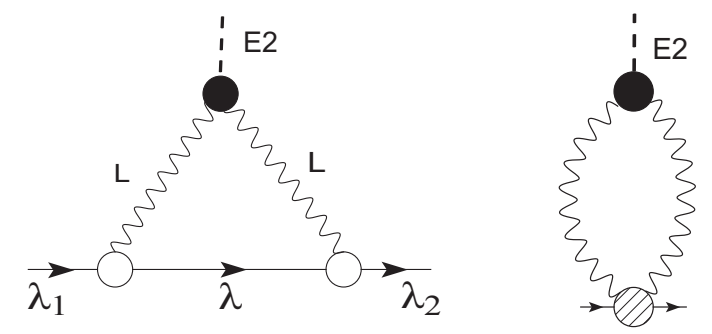

Figure 3. Diagrams for the PC correction due to the quadrupole moment of the $L$-phonon: the triangle (GDD) diagram (left) and the non-pole one (right).

is the residue of the Green function at the pole $\varepsilon=\varepsilon_{\lambda}$. This renormalization is the main of the end effects.

However, there is another end effect, which originates from the non-diagonal terms $\lambda_{1} \neq \lambda$ of these sums. It can be calculated directly and is usually rather small [24, 26, 31]. However, we retain it for completeness, and represent the "end correction" as the sum:

$$
\delta V_{\lambda \lambda}^{\mathrm{end}}=\delta V_{\lambda \lambda}^{Z}+\left(\delta V_{\lambda \lambda}^{\mathrm{end}}\right)^{\prime}
$$

where

$$
\delta V_{\lambda \lambda}^{Z}=\left(Z_{\lambda}-1\right) V_{\lambda \lambda}
$$

Note that Eqs. (11), (12) correspond to partial summation of the diagrams of Fig. 1, and, hence, contain higher order terms in $g_{L}^{2}$. To be consistent up to the order $g_{L}^{2}$, the $Z$-factors in Eqs. (10) and (12) should be expanded in terms of $\partial \Sigma_{\lambda \lambda}(\varepsilon) / \partial \varepsilon, Z_{\lambda}^{\mathrm{ptb}}=1+\partial \delta \Sigma_{\lambda \lambda}(\varepsilon) / \partial \varepsilon$, with the result

$$
\left(\delta V_{\lambda \lambda}^{Z}\right)_{\mathrm{ptb}}=\left.\frac{\partial \delta \Sigma_{\lambda \lambda}(\varepsilon)}{\partial \varepsilon}\right|_{\varepsilon=\varepsilon_{\lambda}} V_{\lambda \lambda}
$$

The energy derivative of the mass operator (8) can be readily found, and we omit for brevity its explicit expression.

Let us now go to the "triangle" diagram $(G G D)$ displayed on the right part of Fig. 1. Evidently, it describes the effect of the induced interaction $\mathcal{F}_{\text {ind }}$ due to the exchange with the $L$-phonon. Below we write explicitly the corresponding formula for the external field with symmetry $E 2$ :

$$
\delta V_{\lambda \lambda}^{G G D}=(-1)^{j-m}\left(\begin{array}{ccc}
j & 2 & j \\
-m & M & m
\end{array}\right)\left\langle v\left\|\delta V^{G G D}\right\| v\right\rangle
$$

where the notation $\lambda=(v, m)$ is used, with the reduced matrix element

$$
\begin{gathered}
\left\langle v_{0}\left\|\delta V^{G G D}\right\| v_{0}\right\rangle=\sum_{12}(-1)^{L+j_{2}-j_{1}}\left\{\begin{array}{ccc}
2 & j_{0} & j_{0} \\
L & j_{2} & j_{1}
\end{array}\right\} \\
\times\langle 2\|V\| 1\rangle\left\langle 0\left\|\bar{g}_{L}\right\| 2\right\rangle\left\langle 1\left\|g_{L}\right\| 0\right\rangle I_{12}\left(\omega_{L}\right), \\
I_{12}\left(\omega_{L}\right)= \\
\frac{1}{\varepsilon_{1}-\varepsilon_{2}}\left[\frac{n_{1}}{\varepsilon_{0}-\varepsilon_{1}+\omega_{L}}+\frac{1-n_{1}}{\varepsilon_{0}-\varepsilon_{1}-\omega_{L}}\right. \\
\left.-\frac{n_{2}}{\varepsilon_{0}-\varepsilon_{2}+\omega_{L}}-\frac{1-n_{2}}{\varepsilon_{0}-\varepsilon_{2}-\omega_{L}}\right],
\end{gathered}
$$


where $\bar{g}_{L}(\omega)=g_{L}(-\omega)$,

$$
\bar{g}_{L}(\mathbf{r} ; \omega)=g_{L 0}(r ; \omega) T_{L L 0}(\mathbf{n}, \alpha)-g_{L 1}(r ; \omega) T_{L L 1}(\mathbf{n}, \alpha) .
$$

In Eqs. (15), (16) the short notation is used: $1=v_{1}, \ldots$

Let us now turn to the diagrams of Fig. 3 describing the contribution of the phonon quadrupole moment. In both of them, the black blob means the phonon quadrupole moment $Q_{L}^{\mathrm{ph}}$. The left one is the usual $(G D D)$ triangle, whereas the right diagram is a non-pole counterpart of the left one, similar to the second diagram in Fig. 2. Contrary to the latter, the non-pole diagram in Fig. 3 plays the crucial role in the problem under consideration. After separating the angular variables in the expression for the triangle $(G D D)$, the left diagram in Fig. 3, we obtain

$$
\delta V_{\lambda \lambda}^{G D D}=(-1)^{j-m}\left(\begin{array}{ccc}
j & 2 & j \\
-m & M & m
\end{array}\right)\left\langle v\left\|\delta V^{G D D}\right\| v\right\rangle,
$$

with the reduced matrix element

$$
\begin{aligned}
& <0\left\|\delta V^{G D D}\right\| 0>=\sum_{1}(-1)^{L+j_{0}+j_{1}} Q_{L}^{\mathrm{ph}} \\
& \times \sqrt{\frac{L(L+1)(2 L+1)}{4 \pi}}\left\{\begin{array}{ccc}
j_{0} & 2 & j_{0} \\
L & j_{1} & L
\end{array}\right\}\left\langle 1\left\|g_{L}\right\| 0\right\rangle \\
& \times\left\langle 0\left\|\bar{g}_{L}\right\| 1\right\rangle\left(I_{1}^{(1)}\left(\omega_{L}\right)+I_{1}^{(2)}\left(\omega_{L}\right)\right), \\
& I_{1}^{(1)}\left(\omega_{L}\right)=\frac{1-n_{1}}{\left(\varepsilon_{0}-\varepsilon_{1}-\omega_{L}\right)^{2}}+\frac{n_{1}}{\left(\varepsilon_{0}-\varepsilon_{1}+\omega_{L}\right)^{2}}, \\
& I_{1}^{(2)}\left(\omega_{L}\right)=-\frac{1}{\omega_{L}}\left(\frac{n_{1}}{\varepsilon_{0}-\varepsilon_{1}+\omega_{L}}+\frac{1-n_{1}}{\varepsilon_{0}-\varepsilon_{1}-\omega_{L}}\right) .
\end{aligned}
$$

The second integral (21) reveals a dangerous behavior at $\omega_{L} \rightarrow 0$. The non-pole diagram on Fig. 3 possess a similar singularity [26]:

$$
I^{\text {non-pole }}=\left.\int \frac{d \omega}{2 \pi i} D_{L}(\omega) D_{L}\left(\omega-\omega_{0}\right)\right|_{\omega_{0} \rightarrow 0}=\frac{4}{\omega_{L}} .
$$

Its behavior at $\omega_{L} \rightarrow 0$ is just the same as of the integral $I_{3}^{(2)}$, Eq. (21). This makes it reasonable to suppose that their sum is regular at $\omega_{L} \rightarrow 0$.

Let us denote the corresponding terms of (19) with (20) and (21) as $\delta V_{G D D}^{(1),(2)}$. An ansatz was proposed in the model under discussion how to deal with these two dangerious terms of $\delta V\left[Q_{L}^{\mathrm{ph}}\right]$. It was supposed that the term $\delta V_{G D D}^{(2)}$ and the tadpole one $\delta V_{\text {tad }}$ cancel each other. Such cancelation does take place for the "fictitious" external fields $V_{0}=\mathbf{j}$ and $V_{0}=1$, thus providing the conservation of the total momentum of the system and the total particle number, correspondingly [3]. In addition, this is true in the case of the spurious $1^{-}$phonon. In the result, the total $g_{L}^{2} \mathrm{PC}$ correction to the effective field becomes equal to

$$
\delta V=\delta V_{\mathrm{ptb}}^{Z}+\delta V_{G G D}+\delta V_{G D D}^{(1)}+\delta V_{\mathrm{end}}^{\prime}
$$

The final ansatz for the quadrupole moments with PC corrections is as follows:

$$
\widetilde{V}_{\lambda \lambda}=Z_{\lambda}\left(V+\delta V_{G G D}+\delta V_{G D D}^{(1)}+\delta V_{\text {end }}^{\prime}\right)_{\lambda \lambda}
$$

Just as Eq. (10) vs Eq. (13), the ansatz (24) differs from the prescription of Eq. (23) in terms higher in $g_{L}^{2}$. It corresponds to making "fat" the ends in Fig. 1 and Fig. 3: $|\lambda\rangle \rightarrow \sqrt{Z_{\lambda}}|\lambda\rangle$. 
Table 1. Characteristics of the $2_{1}^{+}$phonons in even Sn isotopes, $\omega_{2}(\mathrm{MeV})$ and $Q\left(2_{1}^{+}\right)(\mathrm{b})$

\begin{tabular}{|c|c|c|c|c|}
\hline$A$ & $\omega_{2}^{\text {th }}$ & $\omega_{2}^{\exp }$ & $Q^{\text {th }}$ & $Q^{\exp }[35]$ \\
\hline 106 & 1.316 & 1.207 & -0.34 & - \\
108 & 1.231 & 1.206 & -0.39 & \\
110 & 1.162 & 1.212 & -0.50 & $0.30(4)$ \\
112 & 1.130 & 1.257 & -0.45 & $-0.09(10)$ \\
114 & 1.156 & 1.300 & -0.28 & $(-) 0.32(3)$ \\
116 & 1.186 & 1.294 & -0.12 & $-0.17(4)$ \\
118 & 1.217 & 1.230 & -0.01 & $-0.14(10)$ \\
120 & 1.240 & 1.171 & 0.04 & $+0.02(7)$ \\
122 & 1.290 & 1.141 & 0.01 & $-0.13(10)$ \\
124 & 1.350 & 1.132 & -0.07 & $+0.03(13)$ \\
126 & 1.405 & 1.141 & -0.13 & \\
128 & 1.485 & 1.169 & -0.14 & \\
\hline
\end{tabular}

\section{PC corrected quadrupole moments of In and Sb isotopes}

In this section, we present the results of application of the calculation scheme described in the previous section for the proton-odd neighbors of even Sn isotopes. The self-consistent scheme of solving Eqs. (2) and (4) is described in detail in [12] and [13]. The DF3-a version [9] of the Fayans EDF is used. The excitation energies and quadrupole moments of $2_{1}^{+}$states in even $\mathrm{Sn}$ isotopes, which are ingredients of Eqs. (6)-(24), are presented in Table 1. They are taken from [12] and [34]. The fresh values of the experimental quadrupole moments [35] are used.

Table 2 represents separate PC corrections to quadrupole moments considered in the previous Section. Here, the $Z$-factor, column 4 , is found from its definition (10), whereas the perturbation theory prescription (13) is used for finding the $\delta Q_{\mathrm{ptb}}^{Z}$ values in column 5. Thus, the $\delta Q_{\mathrm{ptb}}$ quantity, Eq. (23), is just the sum of four partial corrections in previous columns. At last, the quantity in the last column is $\delta Q_{\text {ph }}=\tilde{V}-V$ where $\tilde{V}$ is the result of the use of Eq. (24) which is the final prescription of the model we use. We see that two main corrections are those due to the $Z$-factor (column 5 ) and due to the induced interaction (the term $\delta Q_{G G D}$, column 6). They always possess different signs, the sum being significantly less in the absolute value than each of them. Therefore two other "small" corrections are sometimes also important. Note that a technical error happened in calculations of the quantity $\delta Q_{\text {end }}^{\prime}$ in [31], therefore the values in the column 8 in Table 2 differ from their analogs in [31]. However, as far as this correction is very small, all the general results of [31] remained valid.

It is worth to mention that the $Z$-factor values are often about 0.5 which makes the use of the perturbation theory in $g_{L}^{2}$ questionable as the value of $(1-Z)$ is a measure of validity of the $g_{L}^{2}$ approximation. Eq. (24) we use contains higher in $g_{L}^{2}$ terms, but it is just an ansatz. The analysis shows that the $g_{L}^{2}$ approximation in semi-magic nuclei is valid on the average, but often one "dangerous" term appears in Eq. (8) with small energy denominator leading to a big contribution to the value of $(1-Z)$. Simultaneously, the same small denominator contributes, with opposite sign, to Eq. (16) for the induced interaction correction. In the result, two inaccuracies compensate each other partially. However, a more consistent approach should be developed for the small denominator situation. Such development based on the method developed in [27] is in our nearest plans.

The final results are presented in Table 3 and Fig. 4. We see that the PC corrections to quadrupole moments taken into account make agreement with experiment better in most cases. In any case, this is 
Table 2. Various PC corrections, induced by the $2_{1}^{+}$phonon, to the quadrupole moments of odd-proton In and $\mathrm{Sb}$ nuclei. $Q$ is the quadrupole moment without PC corrections [13]. Other notation is explained in the text. All values, except $Z$ (dimentionless), are in $\mathrm{b}$.

\begin{tabular}{|l|c|c|c|c|c|c|c|c|c|}
\hline nucl. & $\lambda$ & $Q$ & $Z$ & $\delta Q_{\mathrm{ptb}}^{Z}$ & $\delta Q_{G G D}$ & $\delta Q_{G D D}$ & $\delta Q_{\mathrm{end}}^{\prime}$ & $\delta Q_{\mathrm{ptb}}$ & $\delta Q_{\mathrm{ph}}$ \\
\hline${ }^{105} \mathrm{In}$ & $1 g_{9 / 2}$ & +0.833 & 0.675 & -0.400 & 0.231 & 0.055 & -0.004 & -0.118 & -0.80 \\
${ }^{107} \mathrm{In}$ & $1 g_{9 / 2}$ & +0.976 & 0.584 & -0.692 & 0.404 & 0.094 & -0.008 & -0.201 & -0.117 \\
${ }^{109} \mathrm{In}$ & $1 g_{9 / 2}$ & +1.113 & 0.573 & -0.826 & 0.487 & 0.128 & -0.009 & -0.221 & -0.126 \\
${ }^{111} \mathrm{In}$ & $1 g_{9 / 2}$ & +1.165 & 0.488 & -1.220 & 0.722 & 0.163 & -0.001 & -0.349 & -0.170 \\
${ }^{113} \mathrm{In}$ & $1 g_{9 / 2}$ & +1.117 & 0.576 & -0.820 & 0.484 & 0.071 & -0.010 & -0.275 & -0.159 \\
${ }^{115} \mathrm{In}$ & $1 g_{9 / 2}$ & +1.034 & 0.609 & -0.662 & 0.389 & 0.026 & -0.008 & -0.255 & -0.155 \\
${ }^{117} \mathrm{In}$ & $1 g_{9 / 2}$ & +0.963 & 0.632 & -0.560 & 0.328 & 0.002 & -0.007 & -0.237 & -0.150 \\
${ }^{119} \mathrm{In}$ & $1 g_{9 / 2}$ & +0.909 & 0.621 & -0.553 & 0.323 & -0.008 & -0.007 & -0.244 & -0.152 \\
${ }^{121} \mathrm{In}$ & $1 g_{9 / 2}$ & +0.833 & 0.639 & -0.465 & 0.271 & -0.002 & -0.006 & -0.208 & -0.132 \\
${ }^{123} \mathrm{In}$ & $1 g_{9 / 2}$ & +0.743 & 0.720 & -0.289 & 0.168 & 0.009 & -0.003 & -0.115 & -0.083 \\
${ }^{125} \mathrm{In}$ & $1 g_{9 / 2}$ & +0.663 & 0.738 & -0.232 & 0.134 & 0.015 & -0.003 & -0.088 & -0.064 \\
${ }^{127} \mathrm{In}$ & $1 g_{9 / 2}$ & +0.550 & 0.800 & -0.138 & 0.079 & 0.012 & -0.001 & -0.049 & -0.039 \\
${ }^{115} \mathrm{Sb}$ & $2 d_{5 / 2}$ & -0.882 & 0.551 & 0.717 & -0.275 & -0.025 & -0.022 & 0.395 & 0.218 \\
${ }^{117} \mathrm{Sb}$ & $2 d_{5 / 2}$ & -0.817 & 0.582 & 0.588 & -0.229 & -0.009 & -0.019 & 0.287 & 0.173 \\
${ }^{119} \mathrm{Sb}$ & $2 d_{5 / 2}$ & -0.763 & 0.602 & 0.504 & -0.198 & -0.001 & -0.019 & 0.285 & 0.169 \\
${ }^{121} \mathrm{Sb}$ & $2 d_{5 / 2}$ & -0.721 & 0.591 & 0.497 & -0.196 & 0.003 & -0.017 & 0.240 & 0.145 \\
${ }^{123} \mathrm{Sb}$ & $1 g_{7 / 2}$ & -0.739 & 0.570 & 0.552 & -0.328 & 0.001 & -0.011 & 0.136 & 0.095 \\
\hline
\end{tabular}

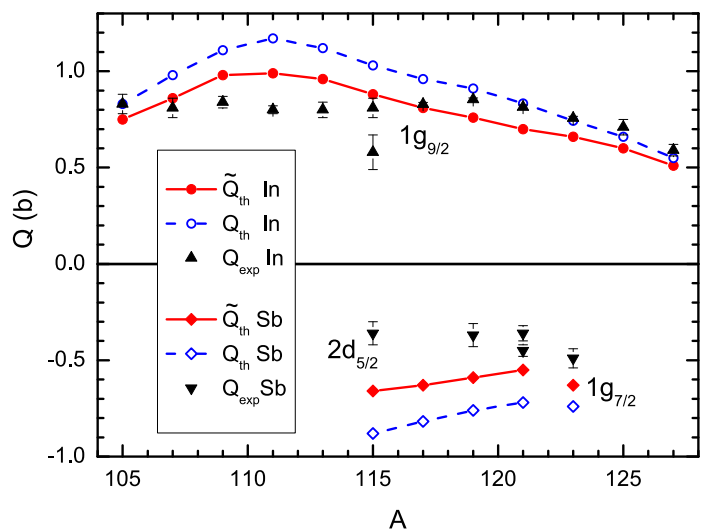

Figure 4. (Color online) Quadrupole moments of odd In and Sb isotopes with and without PC corrections.

true in all the cases where the deviation from experiment of the results without PC corrections is significant, more than $0.1 \mathrm{~b}$, which is evidently a typical accuracy of the theory we develop. For example, this is so for nuclei ${ }^{109,111} \mathrm{In}$ and for all Sb isotopes. The rms value $<\delta \tilde{Q}>_{\mathrm{rms}}=0.16 \mathrm{~b}$ follows from the last column of Table 3 . The corresponding value without PC corrections is significantly bigger, $<\delta Q>_{\mathrm{rms}}=0.27 \mathrm{~b}$. 
Table 3. Quadrupole moments $Q$ (b) of odd In and Sb isotopes. The theoretical values are $Q_{\text {th }}$ and $\tilde{Q}_{\text {th }}$ without and with PC corrections, correspondingly. The differences $\delta Q=Q_{\mathrm{th}}-Q_{\exp }$ and $\delta \tilde{Q}=\tilde{Q}_{\mathrm{th}}-Q_{\exp }$ are given in the last two columns.

\begin{tabular}{|c|c|c|c|c|c|c|}
\hline nucl. & $\lambda$ & $Q_{\text {exp }}$ & $Q_{\text {th }}$ & $\tilde{Q}_{\text {th }}$ & $\delta Q$ & $\delta \tilde{Q}$ \\
\hline${ }^{105} \mathrm{In}$ & $1 g_{9 / 2}$ & $+0.83(5)$ & +0.83 & +0.75 & 0.00 & -0.08 \\
${ }^{107} \mathrm{In}$ & $1 g_{9 / 2}$ & $+0.81(5)$ & +0.98 & +0.86 & 0.17 & 0.05 \\
${ }^{109} \mathrm{In}$ & $1 g_{9 / 2}$ & $+0.84(3)$ & +1.11 & +0.98 & 0.27 & 0.14 \\
${ }^{111} \mathrm{In}$ & $1 g_{9 / 2}$ & $+0.80(2)$ & +1.17 & +0.99 & 0.36 & 0.19 \\
${ }^{113} \mathrm{In}$ & $1 g_{9 / 2}$ & $+0.80(4)$ & +1.12 & +0.96 & 0.32 & 0.16 \\
${ }^{115} \mathrm{In}$ & $1 g_{9 / 2}$ & $+0.81(5)$ & +1.03 & +0.88 & 0.22 & 0.07 \\
& & $0.58(9)$ & & & 0.45 & 0.30 \\
${ }^{117} \mathrm{In}$ & $1 g_{9 / 2}$ & $+0.829(10)$ & +0.96 & +0.81 & 0.136 & -0.02 \\
${ }^{119} \mathrm{In}$ & $1 g_{9 / 2}$ & $+0.854(7)$ & +0.91 & +0.76 & 0.055 & -0.09 \\
${ }^{121} \mathrm{In}$ & $1 g_{9 / 2}$ & $+0.814(11)$ & +0.83 & +0.70 & 0.019 & -0.11 \\
${ }^{123} \mathrm{In}$ & $1 g_{9 / 2}$ & $+0.757(9)$ & +0.74 & +0.66 & -0.014 & -0.10 \\
${ }^{125} \mathrm{In}$ & $1 g_{9 / 2}$ & $+0.71(4)$ & +0.66 & +0.60 & -0.05 & -0.11 \\
${ }^{127} \mathrm{In}$ & $1 g_{9 / 2}$ & $+0.59(3)$ & +0.55 & +0.51 & -0.04 & -0.08 \\
${ }^{115} \mathrm{Sb}$ & $2 d_{5 / 2}$ & $-0.36(6)$ & -0.88 & -0.66 & -0.52 & -0.30 \\
${ }^{117} \mathrm{Sb}$ & $2 d_{5 / 2}$ & - & -0.817 & -0.63 & & - \\
${ }^{119} \mathrm{Sb}$ & $2 d_{5 / 2}$ & $-0.37(6)$ & -0.76 & -0.59 & -0.40 & -0.22 \\
${ }^{121} \mathrm{Sb}$ & $2 d_{5 / 2}$ & $-0.36(4)$ & -0.72 & -0.55 & -0.36 & -0.19 \\
${ }^{123} \mathrm{Sb}$ & $1 g_{7 / 2}$ & $-0.45(3)$ & & & -0.27 & -0.10 \\
${ }^{12} \mathrm{Sb}$ & $-0.49(5)$ & -0.74 & -0.63 & -0.25 & -0.14 \\
\hline
\end{tabular}

Table 4. Characteristics of the $2_{1}^{+}$phonons in even $\mathrm{Pb}$ isotopes, $\omega_{2}(\mathrm{MeV})$ and $Q\left(2_{1}^{+}\right)(\mathrm{b})$

\begin{tabular}{|c|c|c|c|c|}
\hline$A$ & $\omega_{2}^{\text {th }}$ & $\omega_{2}^{\exp }$ & $Q^{\text {th }}$ & $Q^{\exp }[35]$ \\
\hline 202 & 0.823 & 0.960 & -0.15 & - \\
204 & 0.882 & 0.899 & 0.10 & $+0.23(9)$ \\
206 & 0.945 & 0.803 & 0.09 & $+0.05(9)$ \\
208 & 4.747 & 4.086 & 0.05 & $-0.7(3)$ \\
\hline
\end{tabular}

\section{$4 \mathrm{PC}$ corrected quadrupole moments $\mathrm{Bi}$ isotopes}

In this Section, the results are presented of new calculations of the PC corrections, induced by the $2_{1}^{+}$phonons, to quadrupole moments of four odd ${ }^{203-209} \mathrm{Bi}$ isotopes, the odd-proton neighbors of the even ${ }^{202-208} \mathrm{~Pb}$ nuclei. The scheme of presentation of the results is the same as in the previous Section for the odd-proton neighbors of the even tin isotopes. In Table 4, the characteristics of the $2_{1}^{+}$states, which are used in our calculations, are given. Tables 5 and 6 present the separate PC corrections and the results for the PC corrected quadrupole moments, correspondingly. In the Table 4, we see that the excitation energy $\omega\left(2_{1}^{+}\right)$value in the magic ${ }^{208} \mathrm{~Pb}$ nucleus, both experimental and theoretical, is much higher than in the neighboring semi-magic lead isotopes. Such a behavior of the $2_{1}^{+}$levels is typical for magic nuclei, the high value of $\omega\left(2_{1}^{+}\right)$is one of the obligatory characteristics of a magic 
Table 5. Different PC corrections, induced by the $2_{1}^{+}$phonon, to the quadrupole moments of odd-proton Bi nuclei. All notations are the same as in Table 2.

\begin{tabular}{|c|c|c|c|c|c|c|c|c|c|}
\hline nucl. & $\lambda$ & $Q$ & $Z$ & $\delta Q_{\mathrm{ptb}}^{Z}$ & $\delta Q_{G G D}$ & $\delta Q_{G D D}$ & $\delta Q_{\mathrm{end}}^{\prime}$ & $\delta Q_{\mathrm{ptb}}$ & $\delta Q_{\mathrm{ph}}$ \\
\hline${ }^{203} \mathrm{Bi}$ & $1 h_{9 / 2}$ & -1.320 & 0.552 & 1.07 & -0.773 & -0.041 & -0.060 & 0.199 & 0.110 \\
${ }^{205} \mathrm{Bi}$ & $1 h_{9 / 2}$ & -0.954 & 0.736 & 0.342 & -0.247 & 0.012 & -0.020 & 0.087 & 0.064 \\
${ }^{207} \mathrm{Bi}$ & $1 h_{9 / 2}$ & -0.454 & 0.867 & 0.070 & -0.051 & 0.005 & -0.005 & 0.019 & 0.016 \\
${ }^{209} \mathrm{Bi}$ & $1 h_{9 / 2}$ & -0.348 & 0.996 & 0.002 & -0.001 & $0.1 \mathrm{E}-4$ & -0.001 & -0.001 & -0.001 \\
\hline
\end{tabular}

Table 6. Quadrupole moments $Q$ (b) of odd Bi isotopes. All notations are the same as in Table 2 .

\begin{tabular}{|c|c|c|c|c|c|c|}
\hline nucl. & $\lambda$ & $Q_{\exp }$ & $Q_{\text {th }}$ & $\tilde{Q}_{\text {th }}$ & $\delta Q$ & $\delta \tilde{Q}$ \\
\hline${ }^{203} \mathrm{Bi}$ & $1 h_{9 / 2}$ & $-0.93(7)$ & -1.32 & -1.21 & -0.39 & -0.28 \\
& & $-0.67(7)$ & & & -0.65 & -0.54 \\
${ }^{205} \mathrm{Bi}$ & $1 h_{9 / 2}$ & $-0.81(3)$ & -0.95 & -0.89 & -0.14 & -0.08 \\
& & $-0.59(4)$ & & & -0.36 & -0.30 \\
& & $-0.76(2)$ & -0.45 & -0.44 & 0.31 & 0.32 \\
& & & & \\
${ }^{207} \mathrm{Bi}$ & & $-0.55(4)$ & & & 0.10 & 0.11 \\
& & $-0.60(11)$ & & & 0.15 & 0.16 \\
& \multirow{2}{*}{$1 h_{9 / 2}$} & $-0.516(15)$ & -0.348 & -0.349 & 0.168 & 0.167 \\
& & $-0.37(3)$ & & & 0.02 & 0.02 \\
& & $-0.55(1)$ & & & 0.20 & 0.20 \\
& & $-0.77(1)$ & & & 0.42 & 0.42 \\
& & $-0.40(5)$ & & & 0.05 & 0.05 \\
& & $-0.39(3)$ & & & 0.04 & 0.04 \\
\hline
\end{tabular}

nucleus. So high value of the excitation energy means that this state possesses with low collectivity. In the result, the corresponding PC corrections should be very small. The individual role of separate phonons in the PC corrections to the SP energies of the ${ }^{208} \mathrm{~Pb}$ nucleus was examined in [17]. It was found that the lowest of them, the $31_{1}^{-}$phonon, produces the main contribution to the PC correction, about $50 \%$. The other eight phonons (two $5^{-}$, two $2^{+}$, two $4^{+}$, and two $6^{+}$) together give the rest of $50 \%$. Thus, it is obvious from the beginning, that the PC correction to the quadrupole moment of the ${ }^{209} \mathrm{Bi}$ nucleus, induced by the $2_{1}^{+}$phonon, will be negligible. Tables 5 and 6 confirm this. Evidently, the PC correction due to the $3_{1}^{-}$phonon in this case should be much more important. Other general features of the PC corrections to quadrupole moments found in the previous Section for In and $\mathrm{Sb}$ nuclei persist here. We mean the main role of the $\delta Q_{\mathrm{ptb}}^{Z}$ and $\delta Q_{G G D}$ corrections which possess with opposite signs and strongly cancel each other. As to a comparison with experiment, the data presented in the compilation [35] are, unfortunately, too contradictory for each of four Bi isotopes we consider to make definite conclusions.

\section{Conclusions}

The results are presented of the self-consistent calculations of the PC corrections to quadrupole moments of odd semi-magic nuclei on the base of the Fayans EDF DF3-a. The self-consistent model is 
used, developed previously in [24, 26] for magnetic moments and extended for the case of quadrupole moments. The main content of the article consists in the discussion of the results of [31] for the PC corrections, induced by the first $2^{+}$phonon, in the case of the odd In and $\mathrm{Sb}$ isotopes, the odd-proton neighbors of the even tin nuclei. In addition, we present the new results for Bi isotopes, neighboring to the even lead nuclei. The perturbation theory in $g_{L}^{2}$ is used, where $g_{L}$ is the vertex of creating the $L$-phonon.

The main idea of our approach is to refuse from calculation of all terms proportional to $g_{L}^{2}$, as the main their part is taken into account implicitly in the EDF parameters we use. Instead, only such $g_{L}^{2}$ diagrams are separated and calculated explicitly which behave in a non-regular way, i.e. which depend significantly on the nucleus under consideration and the state $\lambda$ of the odd nucleon. Two main PC corrections are the term $\delta Q^{Z}$ connected with the renormalization of the ends of all the PC diagrams due to the phonon $Z$-factor and the one, $\delta Q_{G G D}$, due to the phonon-induced interaction. They always possess opposite signs and cancel each other significantly. Therefore two "small" corrections are also contribute significantly.

For the calculation of one of them, the term $\delta Q_{G D D}$ due to the quadrupole moment of the $L$ phonon, account for the non-pole diagram is of primary importance. As we wrote above, the first $2^{+}$ phonons were taken into account only in this set of calculations. Their quadrupole moments found in [34] were used. The sum of all four PC corrections to quadrupole moments of odd In and Sb isotopes, in most cases, improves agreement with experiment. For the sample of 18 nuclei we consider, the rms value of the difference between the theoretical predictions and experimental values diminishes to $\left\langle\delta \tilde{Q}>_{\text {rms }}=0.16 \mathrm{~b}\right.$ from the value of $\left\langle\delta Q>_{\mathrm{rms}}=0.27 \mathrm{~b}\right.$ for the calculation of [13] without PC corrections.

As to the $\mathrm{Bi}$ isotopes, the experimental data [35] are, unfortunately, too contradictory to make definite conclusions about agreement between them and our theory even at the mean-field level. We hope that the high accuracy of our description of the quadrupole moments of In and Sb isotopes will be a stimulus for experimentalists to clear up the situation with quadrupole moments of Bi isotopes.

Next step we plan includes the contributions to the PC corrections of the first $3^{-}$states. For this aim, their quadrupole moments for the tin and lead isotopes should be first found within the selfconsistent approach developed in [34].

\section{Acknowledgments}

We acknowledge for support the Russian Science Foundation, Grants Nos. 16-12-10155 and 16-1210161.

The work was also partly supported by the RFBR Grant 16-02-00228-a. This work was carried out using computing resources of the federal center for collective usage at NRC "Kurchatov Institute", http://ckp.nrcki.ru.

EES thanks the Academic Excellence Project of the NRNU MEPhI under contract by the Ministry of Education and Science of the Russian Federation No. 02. A03.21.0005.

\section{References}

[1] S.T. Belyaev, Mat.-Fys. Medd. Kgl. Dan. Wid. Selsk., 31, 31 (1959).

[2] V. A. Khodel, E. E. Saperstein, Phys. Rep. 92, 183 (1982).

[3] A. B. Migdal, Theory of finite Fermi systems and applications to atomic nuclei (Nauka, Moscow, 1965; Wiley, New York, 1967).

[4] S. A. Fayans and V. A. Khodel, JETP Lett. 17, 444 (1973). 
[5] A. V. Smirnov, S. V. Tolokonnikov, and S. A. Fayans, Sov. J. Nucl. Phys. 48, 995 (1988).

[6] I. N. Borzov, S. A. Fayans, E. Kromer, and D. Zawischa, Z. Phys. A 355, 117 (1996).

[7] S. A. Fayans, JETP Lett. 68, 169 (1998).

[8] S. A. Fayans, S. V. Tolokonnikov, E. L. Trykov, and D. Zawischa, Nucl. Phys. A 676, 49 (2000).

[9] S. V. Tolokonnikov and E. E. Saperstein, Phys. At. Nucl. 73, 1684 (2010).

[10] I. N. Borzov, E. E. Saperstein, and S. V. Tolokonnikov, Phys. At. Nucl. 71, 469 (2008).

[11] I. N. Borzov, E. E. Saperstein, S. V. Tolokonnikov, G. Neyens, and N. Severijns, Eur. Phys. J. A 45, 159 (2010).

[12] S. V. Tolokonnikov, S. Kamerdzhiev, D. Voytenkov, S. Krewald, and E. E. Saperstein, Phys. Rev. C 84, 064324 (2011).

[13] S. V. Tolokonnikov, S. Kamerdzhiev, S. Krewald, E. E. Saperstein, and D. Voitenkov, EPJA 48, 70 (2012).

[14] S. Kamerdzhiev, S. Krewald, S. Tolokonnikov, E. E. Saperstein, and D.Voitenkov. EPJ Web of Conferences 38, 10002 (2012).

[15] S. V. Tolokonnikov, S. Kamerdzhiev, S. Krewald, E. E. Saperstein and D. Voitenkov. EPJ Web of Conferences 38, 04002 (2012).

[16] J. Terasaki, J. Engel, and G. F. Bertsch, Phys. Rev. C 78, 044311 (2008).

[17] N. V. Gnezdilov, I. N. Borzov, E. E. Saperstein, and S. V. Tolokonnikov, Phys. Rev. C 89, 034304 (2014).

[18] S. V. Tolokonnikov, I. N. Borzov, M. Kortelainen, Yu. S. Lutostansky, and E. E. Saperstein, J. Phys. G 42, 075102 (2015).

[19] S. V. Tolokonnikov, I. N. Borzov, M. Kortelainen, Yu. S. Lutostansky, and E. E. Saperstein, EPJ Web of Conferences, 107, 02003 (2016).

[20] E. E. Saperstein, I. N. Borzov, Yu. S. Lutostansky, and S. V. Tolokonnikov, JETP Lett. 102, 421 (2015).

[21] S. V. Tolokonnikov, I. N. Borzov, Yu. S. Lutostansky, and E. E. Saperstein, Phys. At. Nucl. 79, 21 (2016).

[22] S. V. Tolokonnikov, I. N. Borzov, Yu. S. Lutostansky, I. V. Panov, and E. E. Saperstein, Phys. At. Nucl. 80, 631 (2017).

[23] S. V. Tolokonnikov, I. N. Borzov, M. Kortelainen, Yu. S. Lutostansky, and E. E. Saperstein, EPJA 53: 33 (2017).

[24] E. E. Saperstein, S. Kamerdzhiev, S. Krewald, J. Speth, and S. V. Tolokonnikov, EPL 103, 42001 (2013).

[25] E. E. Saperstein, S. Kamerdzhiev, S. Krewald, J. Speth, and S. V. Tolokonnikov, JETP Lett. 98, 631 (2013).

[26] E. E. Saperstein, S. Kamerdzhiev, S. Krewald, J. Speth, and S. V. Tolokonnikov, Phys. Atom. Nucl. 77, 1033 (2014).

[27] E. E. Saperstein, M. Baldo, S. S. Pankratov, and S. V. Tolokonnikov, JETP Lett. 103, 609 (2016).

[28] E. E. Saperstein, M. Baldo, N. V. Gnezdilov, and S. V. Tolokonnikov, JETP Lett. 103, 1 (2016).

[29] E. E. Saperstein, M. Baldo, N. V. Gnezdilov, and S. V. Tolokonnikov, Phys. Rev. C 93, 034302 (2016).

[30] E. E. Saperstein, M. Baldo, S. S. Pankratov, and S. V. Tolokonnikov, JETP Lett. 103, 743 (2016).

[31] E. E. Saperstein, S. Kamerdzhiev, S. V. Tolokonnikov, and D. Voitenkov, J. Phys. G 44, 065104 (2017).

[32] S. Kamerdzhiev and E. E. Saperstein, EPJA 37, 333 (2008). 
[33] A. Bohr and B. R. Mottelson Nuclear Structure, Vol. 1 (Benjamin, New York, Amsterdam, 1969). [34] D. Voitenkov, S. Kamerdzhiev, S. Krewald, E. E. Saperstein, and S. V. Tolokonnikov, Phys. Rev. C 85, 054319 (2012).

[35] N. J. Stone Table of nuclear magnetic dipole and electric quadrupole moments (IAEA Nuclear Data Section, 2014.) (www-nds.iaea.org/publications/indc/indc-nds-0658) 